\title{
Control of a 3-RRR Planar Parallel Robot Using Fractional Order PID Controller
}

\author{
Auday Al-Mayyahi ${ }^{1} \quad$ Ammar A. Aldair ${ }^{1} \quad$ Chris Chatwin $^{2}$ \\ ${ }^{1}$ Department of Electrical Engineering, College of Engineering, University of Basrah, Basrah, Iraq \\ ${ }^{2}$ Department of Engineering and Design, School of Engineering and Informatics, University of Sussex, Brighton BN1 9RH, UK
}

\begin{abstract}
RRR planar parallel robots are utilized for solving precise material-handling problems in industrial automation applications. Thus, robust and stable control is required to deliver high accuracy in comparison to the state of the art. The operation of the mechanism is achieved based on three revolute (3-RRR) joints which are geometrically designed using an open-loop spatial robotic platform. The inverse kinematic model of the system is derived and analyzed by using the geometric structure with three revolute joints. The main variables in our design are the platform base positions, the geometry of the joint angles, and links of the 3-RRR planar parallel robot. These variables are calculated based on Cayley-Menger determinants and bilateration to determine the final position of the platform when moving and placing objects. Additionally, a proposed fractional order proportional integral derivative (FOPID) is optimized using the bat optimization algorithm to control the path tracking of the center of the 3-RRR planar parallel robot. The design is compared with the state of the art and simulated using the Matlab environment to validate the effectiveness of the proposed controller. Furthermore, real-time implementation has been tested to prove that the design performance is practical.
\end{abstract}

Keywords: 3-RRR planar parallel robot, Cayley-Menger determinants, inverse kinematic model, bilateration, fraction order proportional integral derivate (PID) controller, bat optimization algorithm.

\section{Introduction}

Dexterous movement of robotic manipulators has received significant attention from researchers to enhance the reachable workspace, which can significantly improve industrial automation applications ${ }^{[1]}$. Robotic manipulators can be constructed using different architectures, i.e., serial, parallel, or hybrid manipulators. The first structure is the serial manipulator and this type of robotic manipulator consists of only one serial chain of links. Serial manipulators can be controlled to reach a high degree of freedom based on the number of links. They are widely applied in industrial applications. The second type of manipulators can be designed using a parallel mechanism which exhibits some advantages in terms of dynamic response and accuracy. Furthermore, parallel manipulators can be utilized for applications that need high speed, accuracy, and stiffness ${ }^{[2]}$. Finally, a hybrid manipulator ${ }^{[3]}$ can be constructed based on a combination of serial and parallel manipulators or multiple parallel manipulators connected in sequence. Other hybrid configurations can be created based on different industrial requirements. Such configurations can be utilized to change the end-effector mobility according to the changes in local kinemat-

\footnotetext{
Research Article

Manuscript received January 8, 2020; accepted August 14, 2020; published online November 5, 2020

Recommended by Associate Editor Xun Xu

(C) The Avethor(s) 2020
}

ics. Examples of such configurations are 3-URU (universal revolute universal) and 3-SRU (spherical revolute universal) ${ }^{[4]}$.

Parallel manipulators are closed-loop mechanisms that can be equipped with revolute or prismatic actuators. Such manipulators comprise separate serial chains in which a fixed base is connected to a moving platform. Robust construction is used in such a structure so that it can move bodies of large dimensions with high values of both velocities and accelerations ${ }^{[5]}$. Parallel manipulators can be classified into many types discussed in the survey [6-8] and the structure of such manipulators can be constructed based on 3-RRR and 3-RPR planar parallel robots. The abbreviation of "R" stands for revolute and "P" stands for prismatic. This decides how the mechanism of links will be actuated to make the required movements. The new mechanism of parallel robots can provide highly flexible and accurate movements to obtain different configurations based on solving the inverse position problem. Furthermore, the singularity loci of planar parallel manipulators with revolute joints is solved $[9,10]$.

In the literature survey, many approaches have been reported to derive, analyze, and control inverse kinematics of various models ${ }^{[1-15]}$. In [16], position control of a 3RRR planar parallel robot was presented based on a predictive control system model. The entire design is introduced as a semi-closed loop system. The robotic platform is driven using three direct-current (DC) motors. Each motor is equipped with an encoder to measure the angu- 
lar velocities of the motors. The encoder signals are supplied to the controller as inputs to generate the signals that are used to drive the DC motors. The results obtained demonstrated that the required position of the manipulator was fulfilled.

In [17], a hybrid controller was introduced to control the position of a $3 R R R$ trajectory. This hybrid controller comprises two components, i.e., force and position controllers. Based on the current location of the end-effector in a given free space, the two-hybrid controllers operate simultaneously to control the trajectory of the manipulator in constrained environments. In [18], the development of a 3-RRR planar parallel robot is discussed. This development is based on proposing a new technique that is capable of moving the platform's mass based on a dynamic substitution of the parallel manipulator by three concentrated masses. Such a design allows the system to be transformed from a transformation problem of a reactionless manipulator to a problem of balancing pivoted legs that are carrying concentrated masses.

In [19], the accuracy of a 3RRR parallel robot was improved using a new control method by using extra sensors in the construction of passive joints. The control method was approached initially by performing a sensitivity analysis to analyze the effectiveness and robustness of the proposed method. Later on, the results are proven by using a group of analytic results of the 3-RRR planar parallel robot's performance in the presence of parameter uncertainties. In [20], the kinematics of the 3-RRR planar parallel robot was established based on a kinematic coefficient method. The parallel mechanisms including velocity and acceleration indices were presented and according to such performance indices, the $3 R R R$ planar parallel robotic platform was studied depending on the performance of kinematic mechanisms, its performance depends on the relationship between the design parameters and kinematic performance.

In [21], a 3-RRR planar parallel robotic platform was introduced based on inverse kinematic modeling in which a physical simulation system was conducted by using a Matlab programming environment assuming the end-effector's conditions are known. The movement of the driving element of the robotic system was reached and visualized to demonstrate the process effectiveness of the system. In [22], direct kinematic solutions to a three-degree of freedom (3DOF) planar parallel robotic platform were presented. Also, the derivation of polynomials for both the $3-R R R$ and $3-R P R$ mechanisms were introduced. It demonstrates the differences and similarities between the common configurations of the $3 \mathrm{DOF}$ planar parallel robotic platform.

The motivation for such works comes from the need to rapidly accurately position the robot for grasping and placing especially in industrial automation operations. This can be achieved by reducing the tracking error of 3 -RRR planar parallel robots when following a given path. Although researchers have intensively studied the 3 -RRR in the state of the art, there is still scope to develop and control the 3-RRR planar parallel robot whose mechanical simplicity makes the designed system capable of solving locomotion problems to produce highly flexible automation for industry. The novelty of this paper can be explained by firstly introducing a new algorithm, which has been developed to control the locomotion of a 3-RRR planar parallel robot based on Cayley-Menger determinants and bilateration which has been successful in solving material-handling problems. This has been achieved based on inverse kinematics of the 3-RRR planar parallel robot. Secondly, a fractional-order PID controller has been proposed to control the path tracking of such a platform and the design has been compared with the state of the art which has been published recently. Thirdly, the architecture of the 3RRR planar parallel robotic platform has been designed using 3D Solidworks to prototype $3 R R R$ components based on a $3 \mathrm{D}$ printer and then combined with electronic circuits to conduct some realtime experiments. The contributions of our paper can be emphasized and understood by introducing a new topology based on a new mathematical model that allows bidirection control to reach the same position and this exhibits more flexibility in industrial automation. Furthermore, proposing a new control methodology based on the fractional order proportional integral deviative (FOPID) controller and based on the development of embedded systems, we utilize a new application based on a Polou Maestro Control Centre. This includes an individual control for each servo for the entire movement of the platform to its final position. We sent commands from a PC to this microcontroller, which is also a new feature applied in our design. This increases the flexibility of industrial robots based on a low-cost robotic system for manufacturing tasks such as precision positioning, pick-andplace, and sorting.

The rest of the paper is structured as follows: Section 2 presents Cayley-Menger determinants and bilateration. In Section 2, the proposed algorithm for the 3-RRR planar parallel robot is introduced based on the inverse kinematics model. The structure of the proposed fractional order PID controller along with the stability analysis is introduced in Section 4. The bat optimization algorithm is introduced in Section 5. The simulation results of the developed 3RRR planar parallel robot are produced using the Matlab software as demonstrated in Section 6 . Section 7 describes the real-time experiments that have been completed. Section 8 draws some conclusions.

\section{Cayley-Menger determinants and bilateration}

A Euclidean metric formula has been proposed in the form of a system of polynomial equations. The polynomials can be expressed using the following matrix. This matrix represents a determinant of sequences of points 
$P_{a 1}, \cdots, P_{a n}$ and $P_{b 1}, \cdots, P_{b n}$ and its geometric interpretation represents an important element of Euclidean geometry. This is the well-known as the Cayley-Menger determinant ${ }^{[23-25]}$.

$$
\begin{aligned}
& D\left(a_{1}, \cdots, a_{n} ; b_{1}, \cdots, b_{n}\right)= \\
& 2\left(\frac{-1}{2}\right)^{n}\left|\begin{array}{cccc}
0 & 1 & \cdots & 1 \\
1 & s_{a_{1}, b_{1}} & \cdots & s_{a_{1}, b_{n}} \\
\vdots & \vdots & & \vdots \\
1 & s_{a_{n}, b_{1}} & \cdots & s_{a_{n}, b_{n}}
\end{array}\right| .
\end{aligned}
$$

The distance between $P_{a}$ and $P_{b}$ is squared and can be introduced as $D(a, b)$ and the area of $\Delta P_{a} P_{b} P_{c}=Z_{a, b, c} P_{a, b}$ as $\pm \frac{1}{2} \sqrt{D(a, b, c)}$ which is expressed as positive if $P_{c}$ is to the left side of the vector $P_{a, b}$, and negative otherwise. It can also be proven that $D\left(a_{1}, a_{2} ; b_{1}, b_{2}\right)$ is equivalent to the scalar product between the two vectors, i.e., $P_{a_{1}, a_{2}}$ and $P_{b_{1}, b_{2}}$.

Hence,

$$
\cos (\theta)=\frac{D(a, b ; a, c)}{\sqrt{D(a, b) D(a, c)}}
$$

where $\theta$ is the angle between $P_{a, b}$ and $P_{a, c}$.

The bilateration problem is a geometric problem that can be expressed based on Cayley-Menger determinants. To find feasible locations of a point, e.g., $P_{c}$, assume its distances to two other points, e.g., $P_{a}$ and $P_{b}$, whose locations are known as shown in Fig. 1(a). The projected vectors of those points are shown in Fig. 1(b).

Hence, the orthogonal projection of the position vector, i.e., $P_{c}$ onto $P_{a} P_{b}$ can be expressed as follows:

$$
P=P_{i}+\sqrt{\frac{D(a, c)}{D(a, b)}} \cos \theta P_{a, b}=P_{a}+\frac{D(a, b ; a, c)}{D(a, b)} P_{a, b} .
$$

Additionally, the position vector of $P_{c}$ can be formulated as

$$
P_{c}=P \pm \sqrt{\frac{D(a, b, c)}{D(a, b)}} S P_{a, b}
$$

where $S=\left[\begin{array}{cc}0 & -1 \\ 1 & 0\end{array}\right]$ and the $\pm \operatorname{sgn}$ indicates two (a) Geometric problem

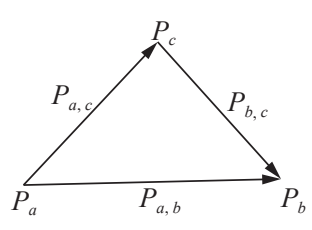

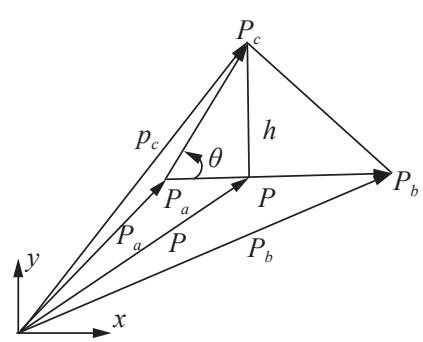

(b) Vectors projection
Fig. 1 Bilateration mirror-symmetric locations of $P_{c}$ with respect to the line defined by the vector $\overline{P_{a} P_{b}}$. By substituting (3) into (4), (5) can be obtained as

$$
P_{c}=P_{a}+\frac{D(a, b ; a, c)}{D(a, b)} P_{a, b} \pm \sqrt{\frac{D(a, b, c)}{D(a, b)}} S P_{a, b} .
$$

Thus,

$$
\begin{aligned}
P_{a, c}= & \frac{D(a, b ; a, c)}{D(a, b)} P_{a, b} \pm \sqrt{\frac{D(a, b, c)}{D(a, b)}} S P_{a, b}= \\
& \frac{1}{D(a, b)}(D(a, b ; a, c) \pm \sqrt{D(a, b, c)} S) P_{a, b} .
\end{aligned}
$$

Equation (6) represents a bilateration problem in terms of Cayley-Menger determinants. Furthermore, another suitable representation can be obtained by expressing this equation in matrix form as

$$
P_{a, c}=Z_{a, b, c} P_{a, b}
$$

where

$$
\begin{aligned}
Z_{a, b, c}= & \frac{1}{D(a, b)}(D(a, b ; a, c) I \pm \sqrt{D(a, b, c)} S)= \\
& \frac{1}{D(a, b)}\left(\begin{array}{cc}
D(a, b ; a, c) & \pm \sqrt{D(a, b, c)} \\
\pm \sqrt{D(a, b, c)} & D(a, b ; a, c)
\end{array}\right) .
\end{aligned}
$$

$Z_{a, b, c}$ is defined as the bilateration matrix and unit matrix (I) is the $2 \times 2$ identity matrix. More expressions can be obtained if the Cayley-Menger determinants involved in $Z_{a, b, c}$ are expanded as

$$
Z_{a, b, c}=\frac{1}{2 s_{a, b}}\left(\begin{array}{cc}
S_{a, b}+S_{a, c}-S_{b, c} & -4 A_{a, b, c} \\
4 A_{a, b, c} & S_{a, b}+S_{a, c}-S_{b, c}
\end{array}\right)
$$

where

$$
\begin{aligned}
& A_{a, b, c}= \\
& \quad \pm \frac{1}{4} \sqrt{\left(S_{a, b}+S_{a, c}+S_{b, c}\right)^{2}-2\left(S_{a, b}^{2}+S_{a, c}^{2}+S_{b, c}^{2}\right)} .
\end{aligned}
$$

$A_{a, b, c}$ is the oriented area of $\Delta P_{a} P_{b} P_{c}$. When it has a positive value, this will cause the chains and their links to move in a specific direction. Whereas, when it has a negative value, the movement will be in the opposite direction. Nonetheless, the center point of the triangular platform will remain fixed.

\section{Inverse kinematics for 3-RRR planar parallel robot}

A geometric description of a 3-RRR planar parallel ro- 
bot is composed of a mobile platform and three RRR serial chains that are joined together at fixed vertices. Each $\mathrm{RRR}$ chain is a serial chain consisting of three rotational joints ${ }^{[26,27]}$. The coordinates of the platform are represented by a point $P_{o}$ that has three coordinates, i.e., $x, y$, and $\theta$, the point $P_{o}(x, y, \theta)$ represents the end-effector position in the working environment of the moving platform at each moment and its orientation. Fig. 2 shows the topology of the $3 \mathrm{RRR}$ planar parallel robotic platform. The definitions of all the indicated variables are summarized below:

1) $\mathrm{P}_{s 1}, P_{s 2}$ and $P_{s 3}$ are the coordinates of the three servo motors.

2) $P_{1}, P_{2}$ and $P_{3}$ are the coordinates of the triangle's vertices.

3) $L_{1}=40 \mathrm{~cm}$ and $L_{2}=40 \mathrm{~cm}$ are the length of the links of each chain.

4) $J_{1}, J_{2}$ and $J_{3}$ are the coordinates of middle points that separate the links of each chain.

5) $P_{\mathrm{o}}$ is the center coordinate of the triangle platform.

6) $T=6 \mathrm{~cm}$ is the distance between a vertex of the triangle and the center coordinate of the triangle.

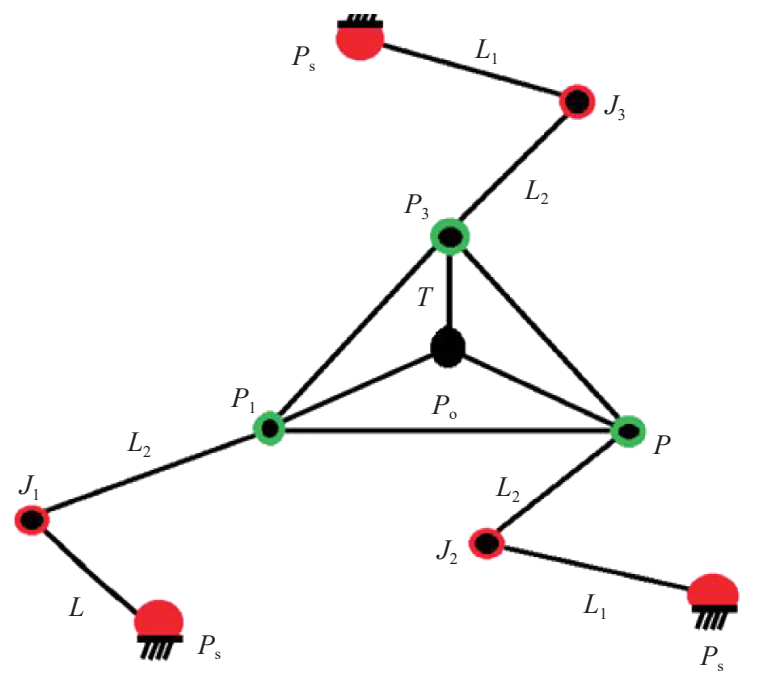

Fig. 2 Topology of 3-RRR planar parallel robot

The derivation of the inverse kinematics equation can be given from the loop closure of the 3-RRR planar parallel robot. Fig. 3 illustrates the end effector triangle platform in 2D based on the $X$ and $Y$ axes. The vertices of the triangular platform are dynamic and the main equations are used for determining the position of each vertex simultaneously. From the center point of the triangle platform, three orientations can be found that are utilized to calculate the movement of each vertex point.

$P_{\mathrm{o}}=$ The center point of the triangle $=(x, y, \theta)$.

$$
\begin{aligned}
& P_{1 x}=x+T \cos \left(\theta+\frac{7 \pi}{6}\right) \\
& P_{1 y}=y+T \sin \left(\theta+\frac{7 \pi}{6}\right)
\end{aligned}
$$

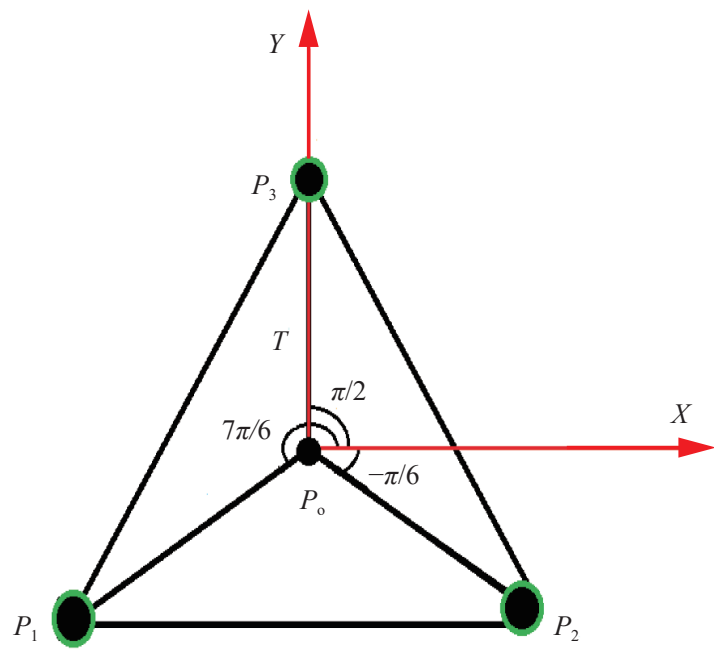

Fig. 3 Topology of the end effector in 2D

$$
\begin{aligned}
& P_{2 x}=x+T \cos \left(\theta-\frac{\pi}{6}\right) \\
& P_{2 y}=y+T \sin \left(\theta-\frac{\pi}{6}\right) \\
& P_{3 x}=x+T \cos \left(\theta+\frac{\pi}{2}\right) \\
& P_{3 y}=y+T \sin \left(\theta+\frac{\pi}{2}\right) .
\end{aligned}
$$

After calculating the triangle vertices points, it is needed to calculate the joint coordinates between the links of each chain. This can be achieved by using a bilateration matrix ${ }^{[28,29]}$. Based on Fig. 4 , the first vertex of the triangle platform, $P_{1}=\left(P_{1 x}, P_{1 y}\right)$ is calculated by $(11)$ and (12). The coordinates of the first servo $P_{s 1}=\left(P_{s 1 x}\right.$, $\left.P_{s 1 y}\right)$ are assumed to be at the original point $(0,0)$.

$L=S_{a, c}=$ Distance between two $P_{1}$ and $P_{s 1}=$

$$
\begin{gathered}
\sqrt{\left(P_{1 y}-P_{s 1 y}\right)^{2}+\left(P_{1 x}-P_{s 1 x}\right)^{2}} \\
{\left[\begin{array}{c}
P_{J 1 x} \\
P_{J 1 y}
\end{array}\right]=\left[\begin{array}{c}
P_{s 1 x} \\
P_{s 1 y}
\end{array}\right]+Z_{a, b, c}\left(\left[\begin{array}{c}
P_{1 x} \\
P_{1 y}
\end{array}\right]-\left[\begin{array}{c}
P_{s 1 x} \\
P_{s 1 y}
\end{array}\right]\right) .}
\end{gathered}
$$

The calculations of the angles that generate the movements of the links in a chain can be derived based on the application of the trigonometry formulas. The notation of the angles $(\varnothing, \varepsilon, \alpha)$ is used as shown in Fig. 5. Based on the Pythagorean theorem, the angles $\varnothing$ and $\alpha$ can be obtained.

$$
\begin{aligned}
& \varnothing=\tan ^{-1}\left(\frac{P_{1 y}}{P_{1 x}}\right) \\
& \alpha=\tan ^{-1}\left(\frac{P_{1 y}-J_{1 y}}{P_{1 x}-J_{1 x}}\right) .
\end{aligned}
$$

The last angle, $\varepsilon$, can be determined by using the well- 


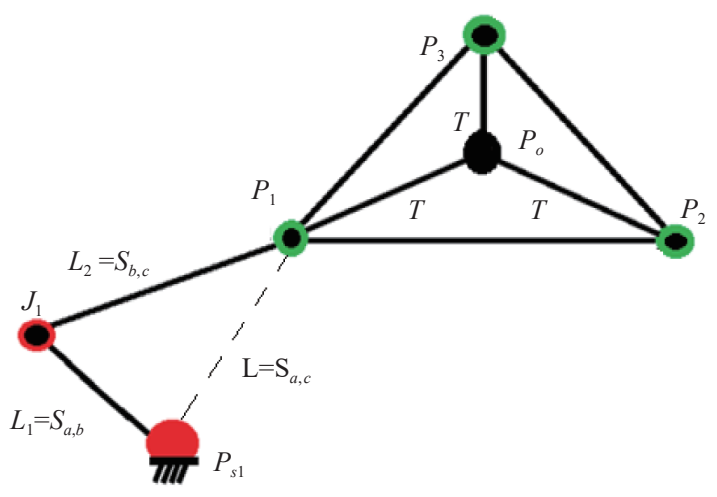

Fig. 4 3-RRR planar parallel robot bilateration diagram

known cosine formula.

$$
\varepsilon=\cos ^{-1} \frac{L_{1}^{2}+L^{2}-L_{2}^{2}}{2 L_{1} L}
$$

After finding the three required angles $(\varnothing, \varepsilon, \alpha)$, we can work in reverse based on the forward kinematics to verify and validate the coordinates of the joint point between the two links of the first chain using the equations below:

$$
\begin{aligned}
& J_{1 x}=L_{1} \cos (\varnothing+\varepsilon) \\
& J_{1 y}=L_{1} \sin (\varnothing+\varepsilon) \\
& P_{1 x}=J_{1 x} \pm L_{2} \cos (\alpha) \\
& P_{1 y}=J_{1 y} \pm L_{2} \sin (\alpha) .
\end{aligned}
$$

Similarly, the coordinates of the other two joints, i.e., $J_{2}$ and $J_{3}$ can be calculated by using the same procedures and equations above. The coordinates of the other two servos are assumed to be fixed at $P_{s 2}(20,0)$ and $P_{s 3}(10,45)$.

\section{Fractional order PID controller}

Many works have been conducted based on the frac-

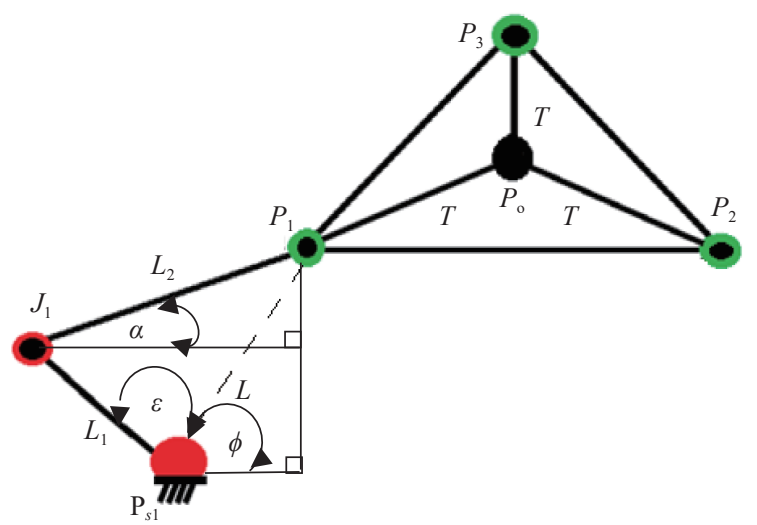

Fig. 5 3-RRR planar parallel robot's trigonometry diagram tional-order PID control in industrial applications in which it has been developed recently to enhance the control performance of the classical PID controller. The fractional-order PID controller can be abbreviated with FOPID and symbolized as $\mathrm{PI}^{\lambda} \mathrm{D}^{\mu}$ controller ${ }^{[30,31]}$. The structure of the FOPID controller consists of five parameters, i.e., proportional gain $K_{p}$, integral gain $K_{i}$, derivative gain $K_{d}$, fractional order of integral $\lambda$, and fractional order of derivative $\mu$ as demonstrated in Fig. 6. As can be observed in this diagram, the transfer function $G(s)$ represents the controlled plant while $R(s)$ and $Y(s)$ are the desired input and the system output, respectively. The disturbance $D(s)$ is also indicated which can be applied externally on the control system. The FOPID controller transfer function $G_{c}(s)$ is supplied by $E(s)$ which represents the error signal between the reference input and the actual output. The control signal $U(s)$ is the action control that is provided to the controlled plan to respond accordingly. The equation that governs the FOPID in the time domain is as

$$
u(t)=K_{p} e(t)+K_{i} D^{-\lambda} e(t)+K_{d} D^{\mu} e(t)
$$

where $e(t)$ and $u(t)$ denote the input and output in the time domain of the controller, respectively.

Based on the Laplace transform, (26) can be rewritten in the frequency domain as

$$
G_{c}(s)=K_{P}+\frac{K_{i}}{s^{\lambda}}+K_{d} s^{\mu} .
$$

The $\mathrm{PI}^{\lambda} \mathrm{D}^{\mu}$ controller is a generalization of the classical PID controller, whose integral and derivative fractional orders values can be given with any arbitrary real number. For instance, if $\lambda=1$ and $\mu=1$, then the FOPID and PID controller are the same. The other three categories of PID controllers, i.e., P, PI, and PD controllers can be obtained when the values of both $(\lambda, \mu)$ equal to $(0,0),(1,0)$ and $(0,1)$, respectively. The relationship of the PID and $\mathrm{PI}^{\lambda} \mathrm{D}^{\mu}$ controllers can be clarified as in $\lambda-\mu$ plane shown in Fig. 7.

The stability is an essential element in designing a control methodology. Hence, study of the stability regions for a fractional order PID controller can be achieved based on the characteristic equation of the controlled system, this is given by

$$
\begin{aligned}
\mathcal{F}(s)= & \kappa\left(K_{i}+K_{p} s^{\lambda}+K_{d} s^{(\lambda+\mu)}\right)+ \\
& \left(1+T_{c} s\right) s^{\lambda} \mathrm{e}^{L s}=\mathfrak{B}(s)+\mathfrak{D}(s) \mathrm{e}^{L s}
\end{aligned}
$$

where $\kappa$ is the steady-state gain of the plant, $T_{c}$ is the time constant of the plant and $L$ is the time delay.

By using the following assumption $\lambda=\frac{\alpha}{\beta}$ and $\mu=\frac{\gamma}{\beta}$, (28) can be rewritten as 


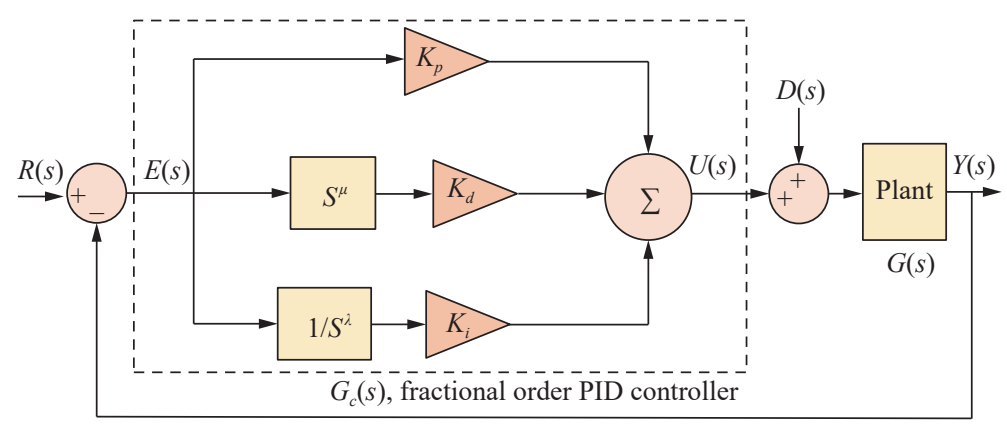

Fig. 6 3RRR planar parallel trigonometry diagram

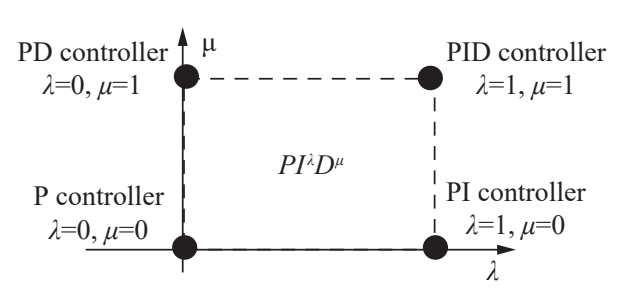

Fig. 7 Generalized FOPID controller

$\mathcal{F}(s)=s^{\frac{\alpha}{\beta}}\left[\kappa K_{d} s^{\frac{\gamma}{\beta}}+\kappa K_{p}+\left(1+T_{c}\right) \mathrm{e}^{L s}\right]+\kappa K_{i}$.

Equation (29) can be rewritten in the $Z$-domain by replacing $s$ with $\frac{z}{L}$ as below:

$\mathcal{F}(z)=\left(\frac{z}{L}\right)^{\frac{\alpha}{\beta}}\left[\kappa K_{d}\left(\frac{z}{L}\right)^{\frac{\gamma}{\beta}}+\kappa K_{p}+\left(1+T_{c}\left(\frac{z}{L}\right)\right) \mathrm{e}^{z}\right]+\kappa K_{i}$

The mapping from $Z$-domain to frequency is performed by replacing each $z$ with $\mathrm{j} \omega$, so that (30) can be rewritten as

$$
\begin{aligned}
\mathcal{F}(\omega)= & \left(\frac{\mathrm{j} \omega}{L}\right)^{\left(\frac{\alpha+\gamma}{\beta}\right)} \kappa K_{d}+\left(\frac{\mathrm{j} \omega}{L}\right)^{\left(\frac{\alpha}{\beta}\right)} \kappa K_{p}+ \\
& \left(\frac{\mathrm{j} \omega}{L}\right)^{\left(\frac{\alpha}{\beta}\right)}\left(1+\mathrm{j} \omega \frac{T_{c}}{L}\right) \mathrm{e}^{\mathrm{j} \omega}+\kappa K_{i} .
\end{aligned}
$$

Using $\left(\mathrm{e}^{\mathrm{j} \omega}=\cos (\omega)+\mathrm{j} \sin (\omega)\right),(31)$ can be rewritten as

$$
\begin{aligned}
& \mathcal{F}(\omega)=\left(\frac{\mathrm{j} \omega}{L}\right)\left(\frac{\alpha+\gamma}{\beta}\right)_{\kappa K_{d}}+\left(\frac{\mathrm{j} \omega}{L}\right)\left(\frac{\alpha}{\beta}\right)_{\kappa K_{p}+} \\
& \left(\frac{\mathrm{j} \omega}{L}\right)\left(\frac{\alpha}{\beta}\right)\left(1+\mathrm{j} \omega \frac{T_{c}}{L}\right) \times \\
& \left(\cos (\omega)-\frac{T_{c}}{L} \omega \sin (\omega)+\mathrm{j} \sin (\omega)+\mathrm{j} \frac{T_{c}}{L} \omega \cos (\omega)\right)+\kappa K_{i} .
\end{aligned}
$$

The characteristic equation of the controller system can be written as a real and imaginary part as

$$
\mathcal{F}(\omega)=\mathcal{F}_{r}(\omega)+\mathrm{j} \mathcal{F}_{i}(\omega)
$$

where

$$
\begin{gathered}
\mathcal{F}_{r}(\omega)=\kappa K_{i}+\kappa K_{d}\left|\operatorname{Re}\left\{(\mathrm{j})\left(\frac{\alpha+\gamma}{\beta}\right)\right\}\right| \omega \mid\left(\frac{\alpha+\gamma}{\beta}\right)\left(\frac{1}{L}\right)^{\left(\frac{\alpha+\gamma}{\beta}\right)}+ \\
\left(\kappa K_{p}+\cos (\omega)-\frac{T}{L} \omega \sin (\omega)\right)\left|\operatorname{Re}\left\{(\mathrm{j})\left(\frac{\alpha}{\beta}\right)\right\}\right||\omega|\left(\frac{\alpha}{\beta}\right)\left(\frac{1}{L}\right)^{\left(\frac{\alpha}{\beta}\right)}- \\
\left(\sin (\omega)+\frac{T_{c}}{L} \omega \cos (\omega)\right)\left|\operatorname{Im}\left\{(\mathrm{j})\left(\frac{\alpha}{\beta}\right)\right\}\right||\omega|\left(\frac{\alpha}{\beta}\right)\left(\frac{1}{L}\right)^{\left(\frac{\alpha}{\beta}\right)_{\operatorname{sgn}(\omega)}}
\end{gathered}
$$

and 


$$
\begin{aligned}
& \mathcal{F}_{i}(\omega)=\kappa K_{d}\left|\operatorname{Im}\left\{(\mathrm{j})\left(\frac{\alpha+\gamma}{\beta}\right)\right\}\right||\omega|\left(\frac{\alpha+\gamma}{\beta}\right)\left(\frac{1}{L}\right)^{\left(\frac{\alpha+\gamma}{\beta}\right)} \operatorname{sgn}(\omega)+ \\
& \left(\kappa K_{p}+\cos (\omega)-\frac{T_{c}}{L} \omega \sin (w)\right)\left|\operatorname{Im}\left\{(\mathrm{j})\left(\frac{\alpha}{\beta}\right)\right)\right||\omega|\left(\frac{\alpha}{\beta}\right)\left(\frac{1}{L}\right)\left(\frac{\alpha}{\beta}\right) \operatorname{sgn}(\omega)+ \\
& \left(\sin (\omega)+\frac{T_{c}}{L} \omega \cos (\omega)\right)\left|\operatorname{Re}\left\{(\mathrm{j})\left(\frac{\alpha}{\beta}\right)\right\}\right||\omega|\left(\frac{\alpha}{\beta}\right)\left(\frac{1}{L}\right)^{\left(\frac{\alpha}{\beta}\right) .}
\end{aligned}
$$

$$
\begin{aligned}
& \mathcal{F}_{r}(\omega)=\kappa\left|\operatorname{Re}\left\{(\mathrm{j})\left(\frac{\alpha}{\beta}\right)\right)\right||\omega|\left(\frac{\alpha}{\beta}\right)\left(\frac{1}{L}\right)^{\left(\frac{\alpha}{\beta}\right)} \times \\
& {\left[K_{p}+\frac{\mid \operatorname{Re}\left\{(\mathrm{j})\left(\frac{\alpha+\gamma}{\beta}\right)\right.}{\left|\operatorname{Re}\left\{(\mathrm{j})\left(\frac{\alpha}{\beta}\right)\right\}\right|}|\omega|\left(\frac{\gamma}{\beta}\right)\left(\frac{1}{L}\right)\left(\frac{\gamma}{\beta}\right) K_{d}+\frac{1}{\kappa}\left|\operatorname{Re}\left\{(\mathrm{j})-\left(\frac{\alpha}{\beta}\right)\right\}\right| \omega \mid-\left(\frac{\alpha}{\beta}\right)\left(\frac{1}{L}\right)^{-\left(\frac{\alpha}{\beta}\right)} K_{i}+\right.} \\
& \left.\frac{1}{\kappa}\left[\left(\cos (\omega)-\frac{T_{c}}{L} \omega \sin (\omega)\right)-\frac{\left|\operatorname{Im}\left\{(\mathrm{j})\left(\frac{\alpha}{\beta}\right)\right\}\right|}{\left|\operatorname{Re}\left\{(\mathrm{j})\left(\frac{\alpha}{\beta}\right)\right\}\right|}\left(\sin (\omega)+\frac{T_{c}}{L} \omega \cos (\omega)\right) \operatorname{sgn}(\omega)\right]\right] .
\end{aligned}
$$

$$
\begin{aligned}
& \left.(-1)^{j} \mathcal{F}_{r}(\omega)\right|_{\omega=\omega_{j}} \gtrless 0 \Rightarrow \\
& (-1)^{j}\left[K_{p}+\frac{\left|\operatorname{Re}\left\{(\mathrm{j})\left(\frac{\alpha+\gamma}{\beta}\right)\right\}\right|}{\mid \operatorname{Re}\{(\mathrm{j})}\left(\frac{\alpha}{\beta}\right)\right\}\left|\omega_{j}\right|\left(\frac{\gamma}{\beta}\right)\left(\frac{1}{L}\right)\left(\frac{\gamma}{\beta}\right) K_{d}+\frac{1}{\kappa}\left|\operatorname{Re}\left\{(\mathrm{j})-\left(\frac{\alpha}{\beta}\right)\right\}\right| \omega_{j} \mid-\left(\frac{\alpha}{\beta}\right)\left(\frac{1}{L}\right)^{-\left(\frac{\alpha}{\beta}\right)} K_{i}+ \\
& \frac{1}{\kappa}\left[\left(\cos \left(\omega_{j}\right)-\frac{T_{c}}{L} \omega_{j} \sin \left(\omega_{j}\right)\right)-\frac{\left|\operatorname{Im}\left\{(\mathrm{j})\left(\frac{\alpha}{\beta}\right)\right\}\right|}{\left|\operatorname{Re}\left\{(\mathrm{j})\left(\frac{\alpha}{\beta}\right)\right\}\right|}\left(\sin \left(\omega_{j}\right)+\frac{T_{c}}{L} \omega \cos \left(\omega_{j}\right)\right) \operatorname{sgn}\left(\omega_{j}\right)\right] \gtrless(-1)^{j+1} K_{p} .
\end{aligned}
$$

Depending on the Hermite-Biehler theorem ${ }^{[32]}$, the necessary and sufficient conditions for the stability of $\mathcal{F}(\omega)$ are:

1) $\mathcal{F}_{r}(\omega)$ and $\mathcal{F}_{i}(\omega)$ have only simple real roots and these are interlaced.

2) $\dot{\mathcal{F}}_{i}(\widehat{\omega}) \mathcal{F}_{i}(\widehat{\omega})-\mathcal{F}_{i}(\widehat{\omega}) \dot{\mathcal{F}}_{r}(\widehat{\omega})>0$, for some $\omega=\widehat{\omega}$ in $(-\infty,+\infty)$.

It is noticeable that if any part of $\mathcal{F}(\omega)$ has only real roots and the interlacing property between the roots of

\section{Springer}

those parts is verified, the other part will also have only real roots. To verify the interlacing property, the value of the $\mathcal{F}_{r}(\omega)$ is computed in the zeros of the $\mathcal{F}_{i}(\omega)$. For $\omega \neq 0$, the $\mathcal{F}_{r}(\omega)$ can be rewritten as (36).

Since $\mathcal{F}_{i}(\omega)$ is an odd function, it always has a root in $\omega=0$ so that

$$
\left.\mathcal{F}_{r}(\omega)\right|_{\omega=0}=\kappa K_{i}
$$


To verify the interlacing property between the roots of the two parts of $\mathcal{F}(\omega)$, one must impose

$$
\left.\mathcal{F}_{r}(\omega)\right|_{\omega=\omega_{0}} \gtrless 0 \Rightarrow K_{i} \gtrless 0
$$

and satisfy (39).

Thus, the stability conditions related to the real part defined a volume in the space of the FOPID parameters.

\section{Bat optimization algorithm}

Among many optimization algorithms introduced in the literature ${ }^{[3-36]}$, the bat algorithm has been introduced and proved to be an effective algorithm that can use the echolocation behavior of bats to optimize given parameters ${ }^{[37-39]}$. A very loud sound pulse can be emitted from bats can, thus, the bats can listen back to the reflected echo from their surroundings. The $i$-th bat flies randomly with given velocity and position, i.e., $v_{i}$ and $p_{i}$ when frequency $f_{\min }$ is fixed. Then, the bat changes its wavelength $\eta$ and loudness $L_{\mathrm{o}}$ to find food. Such behavior can be utilized in searching for the optimal solution so that it can be formulated to optimize the given parameters. The bats' loudness changes from large $L_{\mathrm{o}}$ to small $L_{\min }$ values once they approach their prey.

The new values of frequency, positions and velocities for bats in a given dimensional search space at a specified time step $t$ are defined as in the following equations:

$$
\begin{aligned}
& f_{i}=f_{\min }+\left(f_{\max }-f_{\min }\right) \delta \\
& v_{i}^{t}=v_{i}^{t-1}+\left(p_{i}^{t-1}-p^{*}\right) f_{i} \\
& p_{i}^{t}=p_{i}^{t-1}+v_{i}^{t-1}
\end{aligned}
$$

where the frequency is in a range $\left[f_{\min }, f_{\max }\right]$ and $\delta \in[0,1]$ is the random vector taken from a uniform distribution, and $p^{*}$ is the current global best solution among all $N$ bats. The new position solution based on the current loudness $L_{i}$ of the bat and the maximum allowed variance max (var) during a time stop for a local search can be obtained as

$$
p_{\text {new }}=p_{\text {old }}+\zeta L_{i} \max (v a r)
$$

Once a bat finds the food, the loudness starts decreasing while the rate of pulse emission starts increasing. Therefore, the bat commences maneuvering towards its optimal solution according to the following equation:

$$
L_{i}^{t+1}=\sigma L_{i}^{t}, r_{i}^{t+1}=r_{r}^{o}\left[1-e_{s}^{-\rho t}\right] .
$$

The fitness function of this problem can be found based on the following equation:

$$
F F=\int_{0}^{\infty}\left(w_{1}\left|e_{s}(t)\right|+w_{2} u^{2}(t)\right) \mathrm{d} t+w_{3} t_{u}
$$

The second and the third terms of the above objective function, $\int_{0}^{\infty}\left(w_{2} u^{2}(t)\right) \mathrm{d} t$ and $w_{3} t_{u}$ are utilized to avoid obtaining a large control value and to evaluate the rapidity of rising time response of a control system. Algorithm 1 describes the main steps required for optimizing the parameters of the proposed fractional order PID.

Algorithm 1. Bat algorithm for FOPID controllers

Inputs: Initial bats loudness $r_{i} \in[0.1,0.9]$ and velocities pulse emission rate $r_{i} \in[0,1], \sigma=\rho=0.9$.

Output: optimized parameters $k_{p}, k_{i}$ and $k_{d}, \lambda$ and $\mu$.

1) Loop

2) for $i=1$ to $N$

3) Determine pulse frequency for each bat.

4) Simulate the system and evaluate the performance index FF using (45) and evaluate fitness.

5) Generate new positions for bats by adjusting frequency and updating velocities and positions using (44).

6) Select the bat with the best position and generate a local position around the selected best position of the bat.

7) Randomly generate a new position for a bat.

8) If the position satisfies the conditions.

9) Increase the pulse rate $L_{i}$ and reduce $r_{i}$ using (44).

10) Else go to 7)

11) Rank the bats and determine the best one so far

12) If iteration $<N$

13) go to Step 5)

14) Else

15) Output the best bats.

16) end

17) end

18) end

19) end

The tuned parameters of the FOPID controller optimized by bat optimization algorithm are given in Table 1 .

Table 1 Optimized parameters for the fractional order PID controller using bat algorithm

\begin{tabular}{cccccc}
\hline Parameter & $K_{p}$ & $K_{i}$ & $K_{d}$ & $\lambda$ & $\mu$ \\
\hline Value & 152.8512 & 132.9621 & 8.1254 & 0.4544 & 0.8934 \\
\hline
\end{tabular}

\section{Simulation results}

In this section, two case studies are considered. Firstly, the system is tested without any applied controller in the loop. Thus, it is named as an open-loop system. Secondly, a proposed fractional order PID controller is introduced in the system. Hence, closed-loop system is obtained.

\subsection{Open loop system without controller}

After deriving the equations based on analyzing the 
geometric structure of the inverse kinematic model, a Matlab code was written to obtain the main motions, which verifies the operation and the performance of the kinematic model of the 3-RRR planar parallel robot. Original coordinates have been set up for the three servo motors. The dimensions of the triangle platform and the links are given based on the aforementioned measurements. To validate the operation of the inverse kinematics model, simulation results have been conducted firstly when the $A_{a, b, c}$ is positive and the initial position is placed at $P_{\mathrm{o}}(12,20,0)$ as demonstrated in Fig. 8(a). Secondly, a similar scenario is considered when $A_{a, b, c}$ is negative at the same initial position as shown in Fig. 8(b). The orientation of this point is zero and the $X \& Y$ coordinates are 12, 20 respectively. It has been observed that the same point has been reached using different movement, which has led to manipulation of the links to be opposite to the previous case. This was achieved based on the bilateration problem discussed earlier.

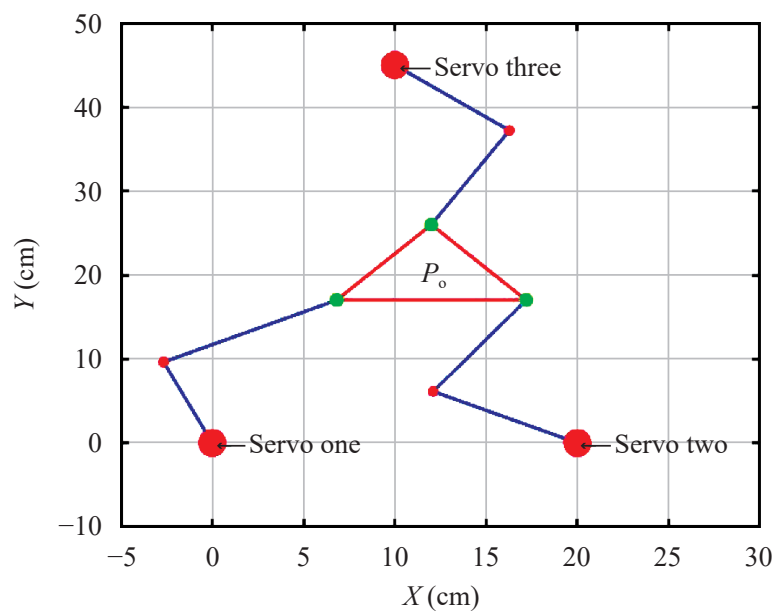

(a) When $A_{a, b, c}$ is positive
Another scenario has been simulated when the destination position of the robotic platform equals to $P_{\mathrm{o}}(12,20, \pi / 6)$. This scenario is similar to the previous one in terms of coordinates. However, the orientation is different which equals to $\pi / 6$. Fig. 9 demonstrates the simulation results of this scenario when $A_{a, b, c}$ is positive as shown in Fig. 9(a). Additionally, Fig. 9(b) depicts the simulation results when $A_{a, b, c}$ is negative.

The final scenario is simulated by changing the position in which both $X$ and $Y$ coordinates have been decreased as well as the orientation in comparing with the previous scenarios. Now, all the three conducted scenarios demonstrate effectively the performance of the developed system that is capable of achieving the required positions feasibly and accurately using two different layouts based on the kinematic characteristics of 3-RRR planar parallel robot in addition to the Cayley-Menger determinants and the bilateration problem.

The final scenario shown in Fig. 10 is simulated by

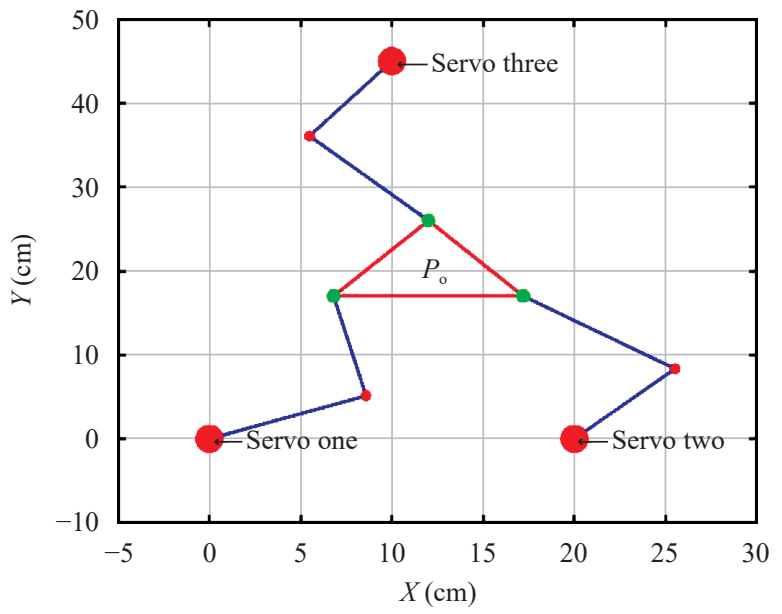

(b) When $A_{a, b, c}$ is negative

Fig. 8 Topology of 3 -RRR planar parallel robot at centre $P_{\mathrm{o}}(12,20,0)$

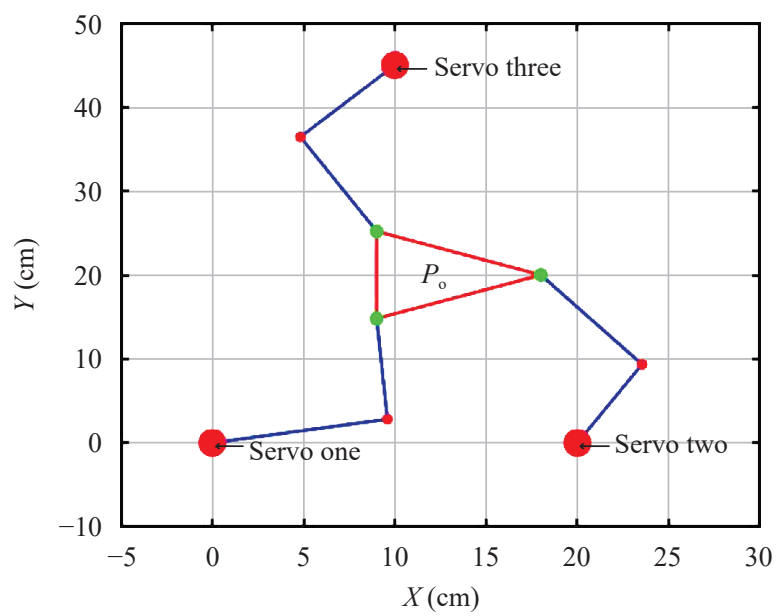

(a) When $A_{a, b, c}$ is positive

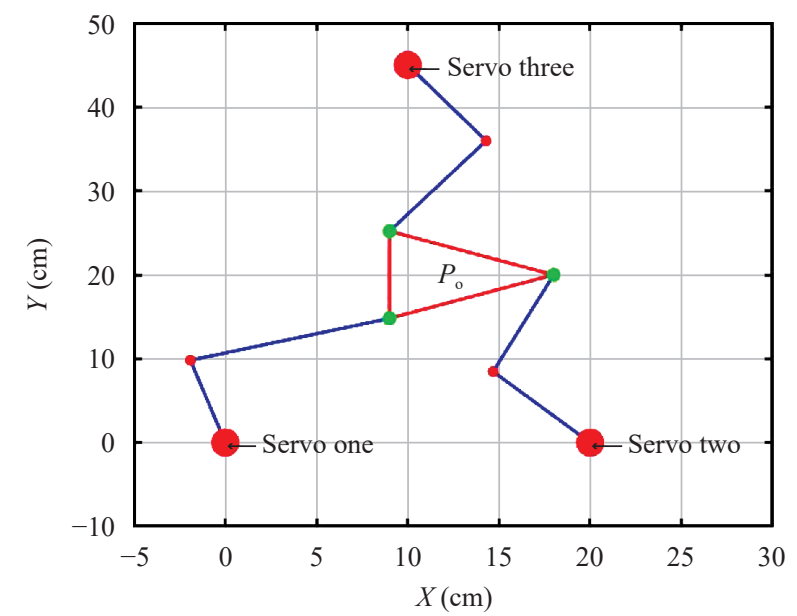

(b) When $A_{a, b, c}$ is negative

Fig. 9 Topology of 3 -RRR planar parallel robot at center $P_{\mathrm{o}}(12,20, \pi / 6)$ 


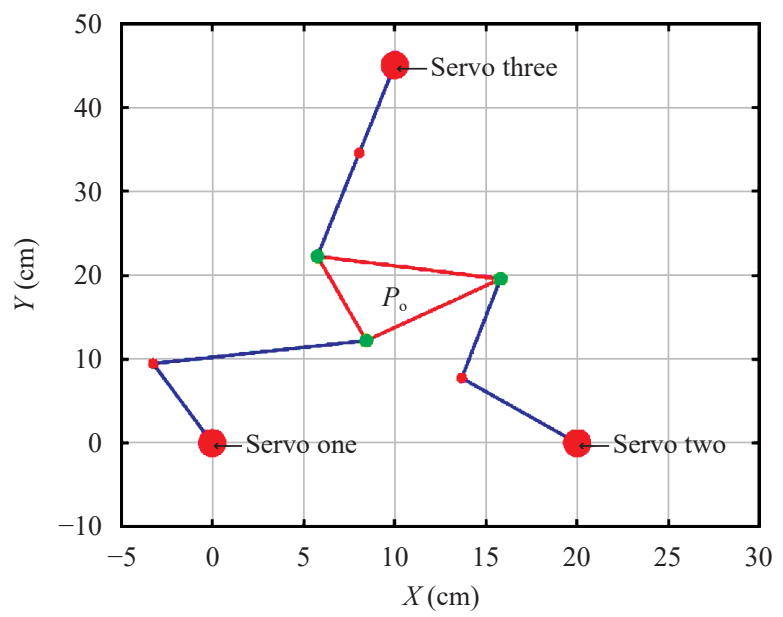

(a) When $A_{a, b, c}$ is positive

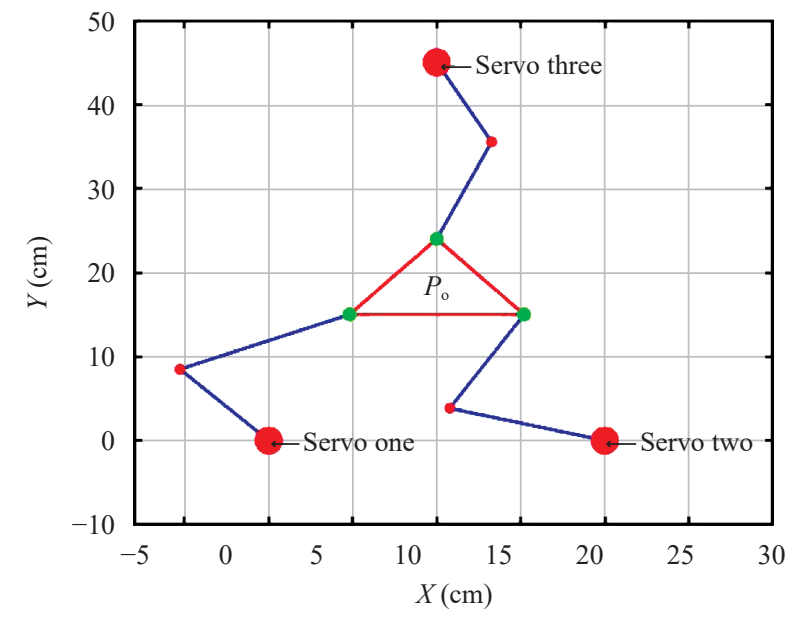

(b) When $A_{a, b, c}$ is negative

Fig. 10 Topology of 3-RRR planar parallel robot at center $P_{\mathrm{o}}(10,18, \pi / 4)$

changing the position in which both $X$ and $Y$ coordinates have been decreased as well as the orientation in comparing with the previous scenarios. Now, all the three conducted scenarios demonstrate effectively the performance of the developed system that is capable of achieving the required positions feasibly and accurately using two different layouts based on the kinematic characteristics of 3RRR planar parallel robot in addition to the CayleyMenger determinants and the bilateration problem.

\subsection{Closed loop system using fractional or- der PID controller}

To investigate the effectiveness and the suitability of the proposed fractional order PID controller, a circular trajectory has been applied to the designed system. Such trajectories are commonly utilized in industrial automation applications. Additionally, to measure the advantages of such a controller in comparing with the state of the art, we have taken the following data which had been already studied as in [40, 41]. Table 2 demonstrates all the numerical values from the state of the $\operatorname{art}^{[40]}$ and our proposed control system. The length of the links $l_{1}=l_{2}=0.4 \mathrm{~m}$, the concentrated mass of the links $m_{g}=1 \mathrm{~kg}$; the concentrated mass of the moving platform $m_{p}=0.2 \mathrm{~kg}$. The desired path equations for $X_{d}$ and $Y_{d}$ coordinates have been stated as in follows where $0 \leq \theta_{d} \leq 360^{\circ}$ :

$$
\left\{\begin{array}{l}
\mathrm{X}_{\mathrm{d}}=0.3-0.3 \cos \left(\theta_{d}\right) \\
\mathrm{Y}_{\mathrm{d}}=0.3 \sin \left(\theta_{d}\right)
\end{array}\right.
$$

The circular path tracking of the traditional PID, the PID tuned by a genetic algorithm (GA), and the FOPID controller optimized by bat algorithm are demonstrated as in Fig. 11. It is observable that the FOPID controller optimized by the bat algorithm delivers a noticeable improvement in terms of response and accuracy. It is ob-
Table 2 Optimized parameters for the fractional order PID controller

\begin{tabular}{cccc}
\hline Parameter & Classic PID & PID with GA & FOPID with bat algorithm \\
\hline$K_{p}$ & 10 & 991.1899 & 152.8512 \\
$K_{i}$ & 100 & 152.2033 & 132.9621 \\
$K_{d}$ & 5 & 9.9589 & 8.1254 \\
$\lambda$ & - & - & 0.4544 \\
$\mu$ & - & - & 0.8934 \\
\hline
\end{tabular}

served that the classic PID controller is tracking further away from the desired path. However, when the PID controller has been optimized by a genetic algorithm, a better response is obtained and it can be noticed clearly that the time response has been significantly improved.

Path tracking errors have been measured to compare the results between the three controllers as shown in Fig. 12. It is noticeable that lowest path error is obtained in case of the FOPID controller optimized by the bat algorithm, where the lowest error equals to $0.6 \mathrm{~mm}$. It is observable that the time response of FOPID controller is approaching the desired path as demonstrated in Fig. 12.

The $X_{f}$ and $Y_{f}$ coordinates obtained for the circular path when the proposed fractional order PID controller has been used are shown in Fig. 13. It is noticed that the movement is feasible and smooth where no spike has been observed in the time response. The angles of the three joints that led the platform to such a movement are demonstrated as in Fig. 14.

To verify that the proposed controller remains stable when an external disturbance is applied to the controlled system, the robustness was investigated. This was achieved by increasing the mass of the end effector to test whether the $3 \mathrm{RRR}$ robot can still move and follow the pre-defined path while carrying additional weight. The optimal values of the FOPID controllers have been fixed to test the effectiveness of the designed controllers when the external disturbance is applied to the controlled sys- 


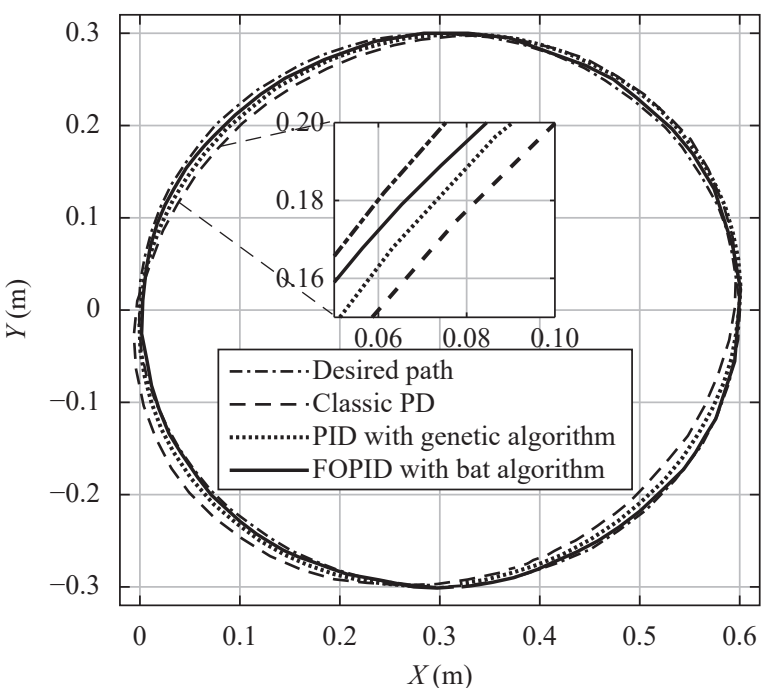

Fig. 11 Circular path tracking of 3-RRR planar parallel robot using classic PID, the PID tuned by GA and FOPID tuned by bat optimization algorithm

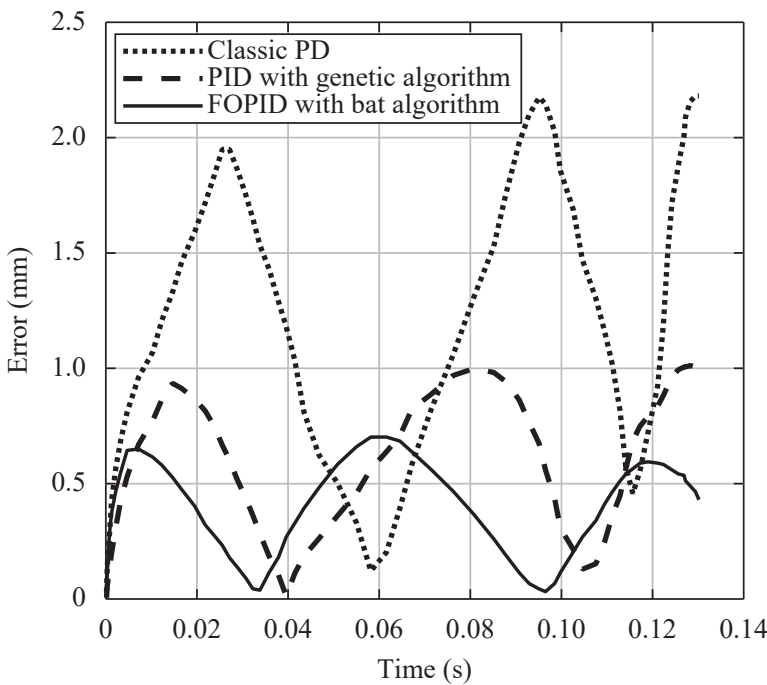

Fig. 12 Path tracking errors of the classic PID, the PID tuned by GA and FOPID tuned by bat optimization algorithm

tem. The value of the added weight equals to $0.6 \mathrm{~kg}$ which is an increase of $50 \%$ of the $3 R R R$ planar parallel robot's weight. It can be seen from Fig. 15 that the platform is still running smoothly along the desired path without a significant difference in path tracking error.

\section{A real-time experiment of 3RRR planer robot}

This section discusses the implementation process of the proposed design based on real-time applications. To achieve this, it is firstly required to prototype the architecture of the 3 -RRR planar parallel robot. This can be conducted using a $3 \mathrm{D}$ printer to implement components designed by Solidworks. Secondly, the embedded system is also needed to provide actuators to guide the move-

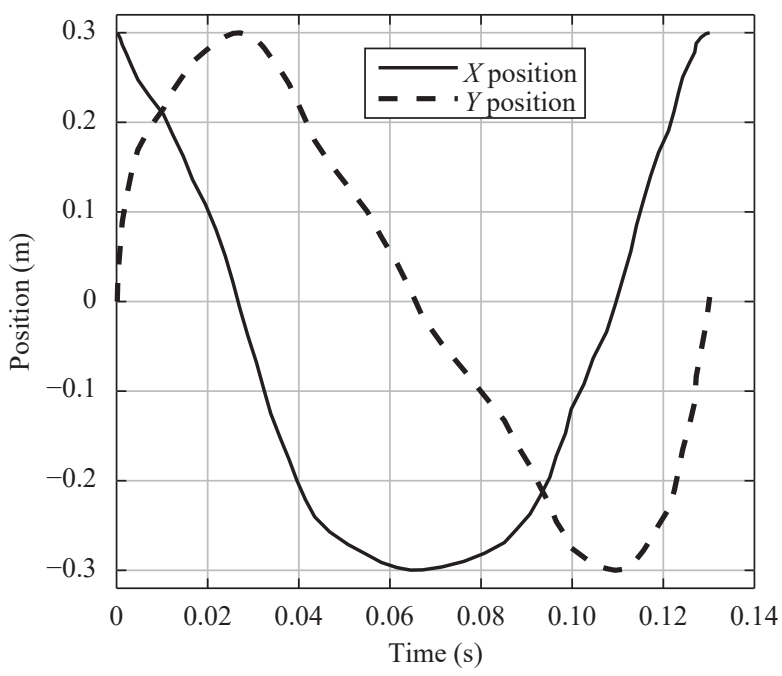

Fig. 13 Actual $X_{f}$ and $Y_{f}$ coordinates of $3 R R R$ platform using FOPID tuned by bat algorithm

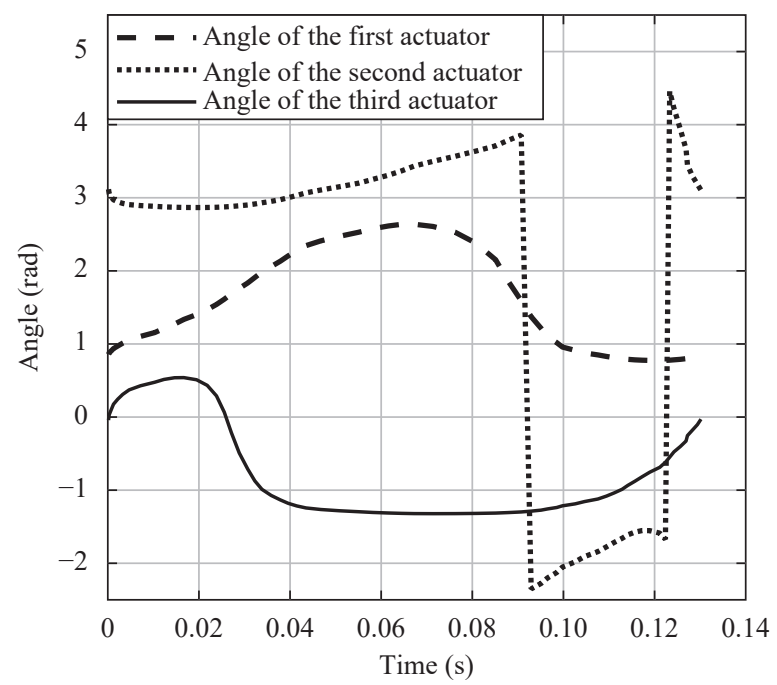

Fig. 14 Angle arms of each base actuator of 3RRR platform using FOPID tuned by bat algorithm

ment of the 3RRR platform as discussed in Sections 7.1 and 7.2 .

\subsection{Solidworks design}

To achieve the practical implementation of the 3-RRR planar parallel robot, the required hardware components are manufactured using a 3D printer. To accomplish this, Solidworks software has been utilized to design and prototype the required components. The main moving base of the platform has been chosen to be a triangle with three holes, each hole is $7 \mathrm{~mm}$ diameter. The thickness of the platform is $6 \mathrm{~mm}$ and distances between the holes are $80 \mathrm{~mm}$. The length of each side equals to $106 \mathrm{~mm}$. The triangle platform was assembled with the middle arm and this arm has two holes, with a diameter equal to $7 \mathrm{~mm}$, which has been designed to connect it with the motor 


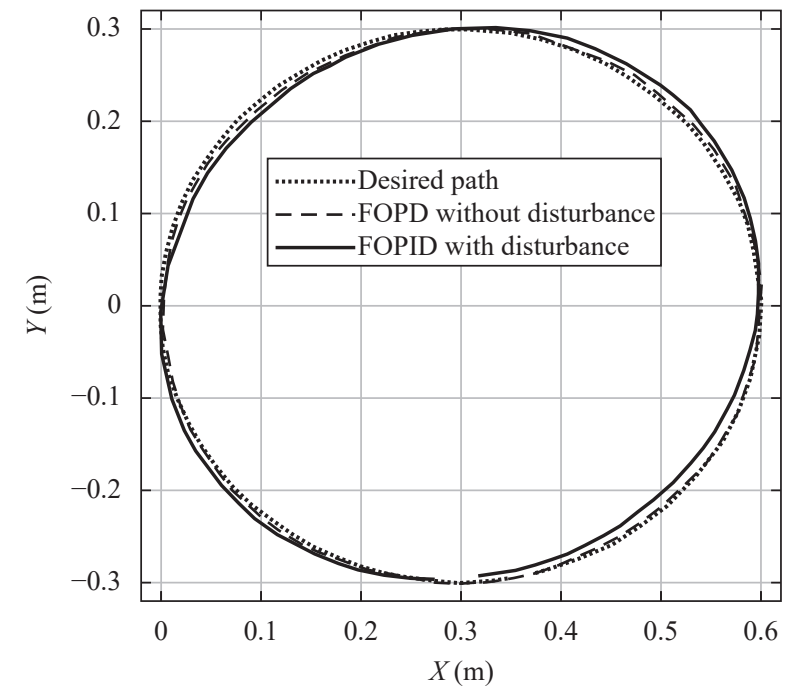

Fig. 15 Circular path tracking of 3RRR platform using FOPID tuned by bat algorithm without and with disturbance

arm and the platform. The length of the arm is $400 \mathrm{~mm}$, the thickness is $5 \mathrm{~mm}$, the width equals to $20.5 \mathrm{~mm}$, and the curve for the edges has a radius of $10 \mathrm{~mm}$. Additionally, the motor arm was designed and assembled with the motor disk and the disk diameter is $20.5 \mathrm{~mm}$. Hence, the width of the arm should be the same or more. The square in the back of the arm has a thickness of $1 \mathrm{~mm}$ and the hole in the middle of the square is $9 \mathrm{~mm}$. There are twelve holes sized $1.9 \mathrm{~mm}$ around it to fix the motor disk to the motor arm. The shape seems to like a clipper from the front with a $7 \mathrm{~mm}$ hole in the middle to connect it with the middle arm. The length of the arm is $400 \mathrm{~mm}$ and width equals to $20.5 \mathrm{~mm}$.

After completing each part successfully, the final 3D parts were combined and assembled. Fig. 16 demonstrates the whole architecture of our design was created using the Solidworks software package.

\subsection{Microcontroller}

The utilized microcontroller is called a Polou Maestro Control Centre, and it can be programmed to control the three servos. It demonstrates the target position of the servo and the speed of the motion. This can be modified manually based on the design requirements and the individual channel of each servo can be set up to limit the minimum and maximum value of any related parameter.

The Maestro's serial mode is set to a universal asynchronous receiver-transmitter UART and it detects the baud rate by default. However, to communicate with it over universal serial bus (USB) via the virtual COM port, it is required to change its setting to one of the two USB modes (USB dual port or USB chained). The baud rate of communication is 9600 bits per second and this is the most commonly used speed that provides reasonable performance for communication.

The generated code based on the proposed sequence of operations can be achieved by sending commands of bytes such as $(132,0,112,46)$. The first byte $(132$, or 0084 in hex) is to specify the set target commands, the second byte (0) is to select which servo channel to control, and the last two bytes (the third and fourth numbers) are specifying the target.

To send a set target command to a different servo channel, we can change the second byte in this command. For instance, this series of numbers $(132,1,32,17)$ is sent. This sets the target of servo channel 2 to the same target value. Alternatively, we can use the set multiple targets to simultaneously set the targets for a contiguous block of channels (e.g., servo channels 0, 4, and 5).

As for commanding our servos to rotate to a specific angle, it depends on the type of servo. Some servos specify the range of travel over a specific pulse width range, e.g., $0-180^{\circ}$, but after testing each servo individually, it was seen that pulse width values correspond to the motion of a slightly different degree, i.e., $0-178^{\circ}$.

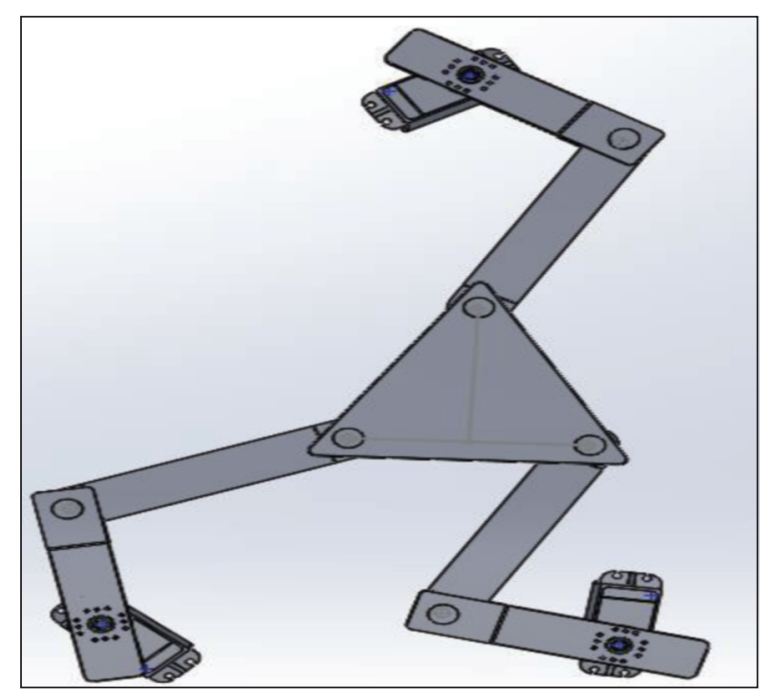

Fig. 16 Architecture of the 3RRR planar parallel robot based on Solidworks

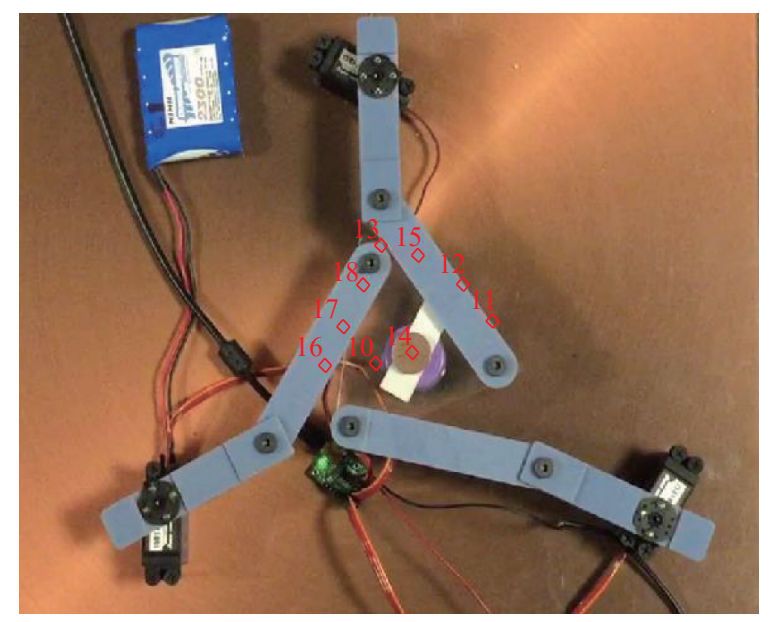

Fig. 17 Hardware of the 3RRR planar parallel robot and its operation 


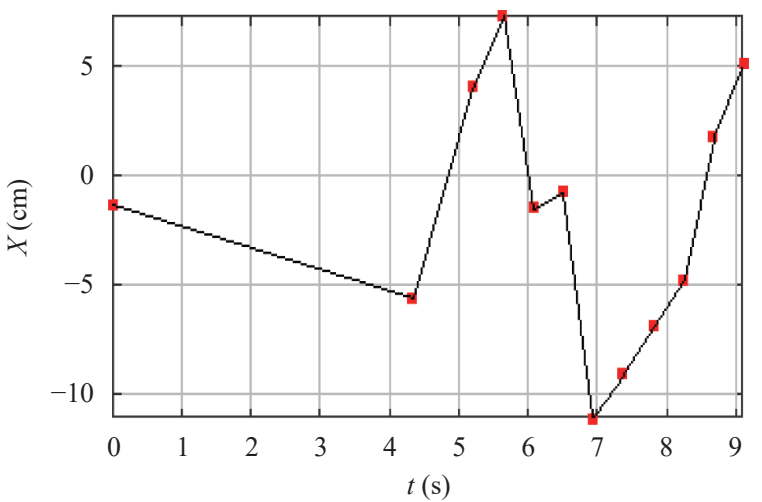

(a) For $X$ axis

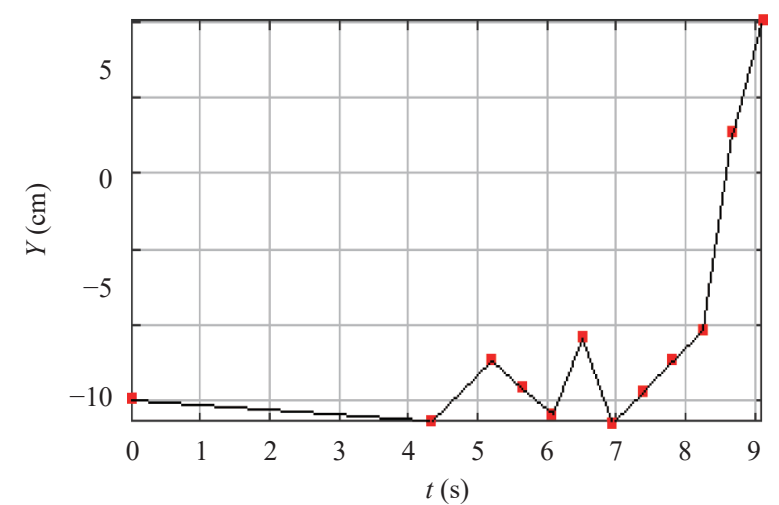

(b) For $Y$ axis

Fig. 18 Time responses of the moving platform

After achieving all the previous requirements successfully, we assembled the mechanical and electrical parts of the 3RRR planar parallel robot platform. Fig. 17 shows the assembly of all parts with each other to produce the final architecture. Finally, we tested real-time movement experimentally as shown in Fig.7, and proved that our design satisfies the operating condition. To demonstrate and validate the movement of our platform, we have demonstrated a tracker to analyze the movement of the platform based on the time responses of the $X$ and $Y$ axes as demonstrated in Figs. 18 (a) and 18 (b), respectively. When the triangle platform moves, the coordinates of $X$ and $Y$ change accordingly within the workspace domain.

\section{Conclusions}

In this paper, the inverse kinematic characteristics of a 3-RRR planar parallel robot have been analyzed and derived based on a specific topology design. The Matlab software was the main simulation environment to achieve programming tasks and to obtain the required results. Several simulation experiments are implemented to verify and validate the functions of the inverse kinematics model using Cayley-Menger determinants and bilateration. The proposed FOPID gave a noticeable improvement in controlling the path tracking ability used on the circular route. It can be observed that the error signal has been decreased. This in turns leads to better accuracy in terms of positioning and placing items especially in precise operations used in industrial automation. After modelling the inverse kinematics, all the dimensions and movements have been satisfied. We have prototyped each component of our design. These components have been 3D printed to construct the final topology of the proposed architecture. Practical experiments were implemented to verify and validate the overall performance of the 3-RRR planar parallel robot. Communications between a computer and the platform were made to send coded signals that represent the movement sequence of the platform. Accordingly, the required movements have been achieved and the operational performance was validated based on real-time scenarios. In all case studies, it was demonstrated that the performance of the developed system is capable of achieving the required positions smoothly and accurately.

\section{Open Access}

This article is licensed under a Creative Commons Attribution 4.0 International License, which permits use, sharing, adaptation, distribution and reproduction in any medium or format, as long as you give appropriate credit to the original author(s) and the source, provide a link to the Creative Commons licence, and indicate if changes were made.

The images or other third party material in this article are included in the article's Creative Commons licence, unless indicated otherwise in a credit line to the material. If material is not included in the article's Creative Commons licence and your intended use is not permitted by statutory regulation or exceeds the permitted use, you will need to obtain permission directly from the copyright holder. To view a copy of this licence, visit http://creativecommons.org/licenses/by/4.0/.

\section{References}

[1] R. Saravanan, S. Ramabalan, C. Balamurugan, A. Subash. Evolutionary trajectory planning for an industrial robot. International Journal of Automation and Computing, vol.7, no. 2, pp.190-198, 2010. DOI: 10.1007/s11633-0100190-8.

[2] O. Hamdoun, L. El Bakkali, F. Z. Baghli. Analysis and optimum kinematic design of a parallel robot. Procedia Engineering, vol.181, pp.214-220, 2017. DOI: 10.1016/ j.proeng.2017.02.374.

[3] G. Coppola, D. Zhang, K. F. Liu. A 6-DOF reconfigurable hybrid parallel manipulator. Robotics and Computer-integrated Manufacturing, vol.30, no.2, pp.99-106, 2014. DOI: 10.1016/j.rcim.2013.09.011.

[4] M. Palpacelli, L. Carbonari, G. Palmieri, M. Callegari. Design of a lockable spherical joint for a reconfigurable 3URU parallel platform. Robotics, vol. 7, no. 3, Article number 42, 2018. DOI: $10.3390 /$ robotics7030042.

[5] L. W. Tsai. Robot analysis: The Mechanics of Serial and Parallel Manipulators, New York, USA: John Wiley \& 
Sons, Inc., 1999.

[6] M. Hu, J. S. Shi. The kinematic analyses of the 3-DOF parallel machine tools. International Journal of Automation and Computing, vol. 8, no. 1, pp.107-111, 2011. DOI: 10.1007/s11633-010-0561-1.

[7] X. M. Niu, G. Q. Gao, X. J. Liu, Z. D. Bao. Dynamics and control of a novel 3-DOF parallel manipulator with actuation redundancy. International Journal of Automation and Computing, vol.10, no.6, pp.552-562, 2013. DOI: 10.1007/s11633-013-0753-6.

[8] M. Mazare, M. Taghizadeh, M. Rasool Najafi. Kinematic analysis and design of a 3-DOF translational parallel robot. International Journal of Automation and Computing, vol. 14, no. 4, pp. 432-441, 2017. DOI: 10.1007/s11633-0171066-y.

[9] I. A. Bonev, C. M. Gosselin. Singularity loci of planar parallel manipulators with revolute joints. In Proceedings of the 2nd Workshop on Computational Kinematics, Seoul, South Korea, 2001.

[10] C. Nasa, S. Bandyopadhyay. Trajectory-tracking control of a planar 3-RRR parallel manipulator with singularity avoidance. In Proceedings of the 13th World Congress in Mechanism and MachineScience, Guanajuato, Mexico, 2011.

[11] I. A. Bonev, S. Briot, P. Wenger, D. Chablat. Changing assembly modes without passing parallel singularities in non-cuspidal 3-RPR planar parallel robots. In Proceedings of the 2nd Workshop on Fundamental Issues and Future Research Directions for Parallel Mechanisms and Manipulators, Montpellier, France, 2008.

[12] S. Staicu. Kinematics of the 3-RRR planar parallel robot. UPB Scientific Bulletin, Series D: Mechanical Engineering, vol. 70, no. 2, pp. 3-14, 2008.

[13] I. A. Bonev, D. Zlatanov, C. M. Gosselin. Singularity analysis of 3-DOF planar parallel mechanisms via screw theory. Journal of Mechanical Design, vol.125, no.3, pp. 573-581, 2003. DOI: $10.1115 / 1.1582878$.

[14] M. Rodelo, J. L. Villa, J. Duque, E. Yime. Kinematic analysis and performance of a planar 3RRR parallel robot with kinematic redundancy using screw theory. In Proceedings of the 2nd IEEE Colombian Conference on Robotics and Automation, IEEE, Barranquilla, Colombia, 2018. DOI: $10.1109 /$ CCRA.2018.8588123.

[15] S. Kucuk. Energy minimization for 3-RRR fully planar parallel manipulator using particle swarm optimization. Mechanism and Machine Theory, vol.62, pp.129-149, 2013. DOI: 10.1016/j.mechmachtheory.2012.11.010.

[16] A. K. Dash, S. Krishnamurthy, S. Prasadh, V. Sundar. Position control of a 3-RRR planar parallel manipulator with non-planar links using external encoders. Advanced Materials Research, vol.971-973, pp.1280-1283, 2014. DOI: 10.4028/www.scientific.net/AMR.971-973.1280.

[17] S. X. Tian, S. Z. Wang. Hybrid position/force control for a RRR 3-DoF manipulator. Applied Mechanics and Materials, vol. 48-49, pp. 589-592, 2011. DOI: 10.4028/www.scientific.net/AMM.48-49.589.

[18] V. H. Arakelian, M. R. Smith. Design of planar 3-DOF 3RRR reactionless parallel manipulators. Mechatronics, vol. 18, no. 10, pp.601-606, 2008. DOI: 10.1016/j.mechatronics.2008.05.002.

[19] A. Zubizarreta, M. Marcos, I. Cabanes, C. Pinto, E. Portillo. Redundant sensor based control of the 3RRR parallel robot. Mechanism and Machine Theory, vol.54, pp.1-17, 2012. DOI: 10.1016/j.mechmachtheory.2012.03. 004 .
[20] F. Q. Chang, Z. Sijun, G. Xijuan. Kinematical performance analysis for planar parallel mechanism 3RRR. Chinese Journal of Mechanical Engineering (English Edition), vol.17, no.S1, pp.181-184, 2004. DOI: 10.3901/ CJME.2004.supp.181.

[21] J. R. Gao, Y. Z. Wang, Z. P. Chen. Modelling and simulation of inverse kinematics for planar 3-RRR parallel robot based on simmechanics. Advanced Materials Research, vol.898, pp. 510-513, 2014. DOI: 10.4028/www.scientific. net/AMR.898.510.

[22] D. Oetomo, H. C. Liaw, G. Alici, B. Shirinzadeh. Direct kinematics and analytical solution to 3RRR parallel planar mechanisms. In Proceedings of the 9th International Conference on Control, Automation, Robotics and Vision, IEEE, Singapore, 2006. DOI: 10.1109/ICARCV.2006.345 064.

[23] F. Thomas, L. Ros. Revisiting trilateration for robot localization. IEEE Transactions on Robotics, vol.21, no.1, pp. 93-101, 2005. DOI: 10.1109/TRO.2004.833793.

[24] C. D'Andrea, M. Sombra. The Cayley-Menger determinant is irreducible for $n \geq 3$. Siberian Mathematical Journal, vol.46, no. 1, pp.71-76, 2005. DOI: 10.1007/s11202-0050007-0.

[25] N. Rojas, F. Thomas. On closed-form solutions to the position analysis of Baranov trusses. Mechanism and Machine Theory, vol.50, pp.179-196, 2012. DOI: 10.1016/j.mechmachtheory.2011.10.010.

[26] R. L. Williams, B. H. Shelley. Inverse kinematics for planar parallel manipulators. In Proceedings of ASME Design Technical Conferences, Sacramento, Calif, USA, 1997.

[27] J. Alvarez-Munoz, N. Marchand, J. F. Guerrero-Castellanos, J. J. Tellez-Guzman, J. Escareno, M. Rakotondrabe. Rotorcraft with a 3DOF rigid manipulator: quaternion-based modeling and real-time control tolerant to multi-body couplings. International Journal of Automation and Computing, vol.15, no. 5, pp. 547-558, 2018. DOI: 10.1007/s11633-018-1145-8.

[28] N. Rojas. Distance-based Formulations for the Position Analysis of Kinematic Chains, Ph. D. dissertation, Polytechnic University of Catalonia, Catalunya, Spain, 2012.

[29] N. Rojas, F. Thomas. The forward kinematics of 3-RPR planar robots: a review and a distance-based formulation. IEEE Transactions on Robotics, vol. 27, no. 1, pp.143-150, 2011. DOI: $10.1109 /$ TRO.2010.2092251.

[30] L. Xu, B. Y. Song, M. Y. Cao, Y. H. Xiao. A new approach to optimal design of digital fractional-order $P I^{\lambda} D^{\mu}$ controller. Neurocomputing, vol.363, pp.66-77, 2019. DOI: 10.1016/j.neucom.2019.06.059.

[31] N. Kumar, B. Tyagi, V. Kumar. Application of fractional order PID controller for AGC under deregulated environment. International Journal of Automation and Computing, vol.15, no. 1, pp.84-93, 2018. DOI: 10.1007/s11633016-1036-9.

[32] R. Bellman, K. L. Cooke. Differential-Difference Equations, London, Britain: Academic Press, 1963.

[33] A. A. Aldair, W. J. Wang. Neural controller based full vehicle nonlinear active suspension systems with hydraulic actuators. International Journal of Control and Automation, vol. 4, no. 2, pp. 79-94, 2011.

[34] A. Al-Mayyahi, W. Wang, P. Birch. Path tracking of autonomous ground vehicle based on fractional order PID controller optimized by PSO. In Proceedings of the 13th IEEE International Symposium on Applied Machine Intelligence and Informatics, IEEE, Herl'any, Slovakia, 
pp. 109-114, 2015. DOI: 10.1109/SAMI.2015.7061857.

[35] A. A. Aldair, E. B. Alsaedee, T. Y. Abdalla. Design of AB$\mathrm{CF}$ control scheme for full vehicle nonlinear active suspension system with passenger seat. Iranian Journal of Science and Technology, Transactions of Electrical Engineering, vol.43, no. 1, pp. 289-302, 2019. DOI: 10.1007/s40998018-0134-9.

[36] A. A. Aldair, A. T. Rashid, M. T. Rashid, E. B. Alsaedee. Adaptive fuzzy control applied to seven-link biped robot using ant colony optimization algorithm. Iranian Journal of Science and Technology, Transactions of Electrical Engineering, vol.43, no.4, pp.797-811, 2019. DOI: 10.1007/ s40998-019-00201-x.

[37] U. Arora, M. E. A. Lodhi, T. K. Saxena. PID parameter tuning using modified BAT algorithm. Journal of Automation and Control Engineering, vol.4, no. 5, pp.347-352, 2016. DOI: 10.18178/joace.4.5.347-352.

[38] M. Rahmani, A. Ghanbari, M. M. Ettefagh. A novel adaptive neural network integral sliding-mode control of a biped robot using bat algorithm. Journal of Vibration and Control, vol. 24, no.10, pp. 2045-2060, 2018. DOI: 10.1177/ 1077546316676734 .

[39] A. Al-Mayyahi, W. J. Wang, P. Birch. LevenbergMarquardt optimised neural networks for trajectory tracking of autonomous ground vehicles. International Journal of Mechatronics and Automation, vol.5, no. 2-3, pp. 140-153, 2015. DOI: 10.1504/IJMA.2015.075960.

[40] L. C. Sheng, W. Li. Optimization design by genetic algorithm controller for trajectory control of a 3-RRR parallel robot. Algorithms, vol.11, no.1, Article number 7, 2018. DOI: $10.3390 / \mathrm{a} 11010007$.

[41] A. Al-Mayyahi, W. J. Wang, P. Birch. Design of fractional-order controller for trajectory tracking control of a nonholonomic autonomous ground vehicle. Journal of Control, Automation and Electrical Systems, vol.27, no.1, pp. 29-42, 2016. DOI: 10.1007/s40313-015-0214-2.

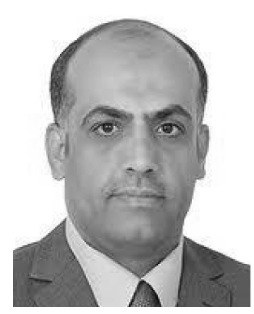

Auday Al-Mayyahi received the B.Sc. and M.Sc. degrees in electrical engineering from University of Basrah, Iraq in 2003 and 2007, respectively. He received the Ph.D. degree in industrial automation from University of Sussex, UK in 2018. Currently, he is a lecturer at University of Basrah in which he has taught different topics within the area of electrical engin- eering. Additionally, he is currently the director of Avicenna Elearning Center at University of Basrah.

His research interests include robotics, automation and intelligent control systems.

E-mail: auday.essa@uobasrah.edu.iq

ORCID iD: 0000-0002-8387-1631

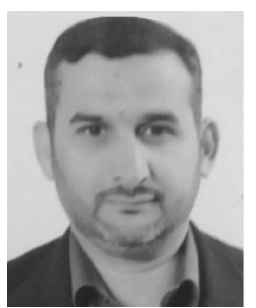

Ammar A. Aldair received the B. Sc. degree in electrical engineering from University of Basrah, Iraq in 2000. He received the M. Sc. degree in control and systems engineering from University of Basrah, Iraq in 2003. He received the Ph.D. degree in control and systems engineering from University of Sussex, UK in 2012. From 2003-2008, he was a lecturer in Electrical Department, University of Basrah, Iraq. He teaches many subjects such as mathematics, logic systems, electrical circuits, electronic circuits, control systems and advance control systems. He is currently an assistance professor at University of Basrah.

His research interest is design of intelligent control systems.

E-mail: mmr.ali2@googlemail.com

ORCID iD: 0000-0002-5220-9848

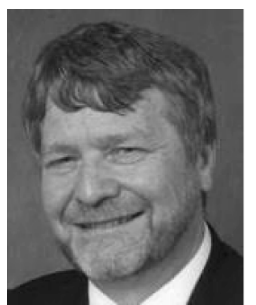

Chris Chatwin holds the Chair of Engineering, University of Sussex, UK. He is Research Director of the IIMS Research Centre and the Laser and Photonics Systems Engineering Group. He has been a member of the University: Senate, Council and Court At Sussex. He has published two research monographs: one on numerical methods, the other on hybrid optical/digital computing, and more than seven hundred international papers. He is on the editorial board of the International Journal Lasers in Engineering. He is also a member of The Institution of Electrical and Electronic Engineers, IEEE Computer Society, the British Computer Society, the Association of Industrial Laser Users, European Optical Society. He is a Chartered Engineer, EuroEngineer, International Professional Engineer, Chartered Physicist, Chartered Scientist and a Fellow of The Institution of Electrical Engineers, The Institution of Mechanical Engineers, The Institute of Physics and The Royal Society for Arts, Manufacture and Commerce.

His research interests include robotics, computer vision, recognition and tracking, surveillance and security systems.

E-mail: c.r.chatwin@sussex.ac.uk (Corresponding author) ORCID iD: 0000-0001-9371-8502 